\title{
ON SOME PARABOLIC-ELLIPTIC SYSTEM WITH SELF-SIMILAR PRESSURE TERM
}

\author{
ROBERT STAŃCZY \\ Instytut Matematyczny, Uniwersytet Wrockawski \\ Pl. Grunwaldzki 2/4, 50-384 Wroctaw, Poland \\ E-mail:stanczr@math.uni.wroc.pl
}

\begin{abstract}
A priori estimates for solutions of a system describing the interaction of gravitationally attracting particles with a self-similar pressure term are proved. The presented theory covers the case of the model with diffusions that obey either Fermi-Dirac statistics or a polytropic one.
\end{abstract}

1. Introduction. We consider the following initial-boundary value problem:

$$
\begin{array}{rll}
n_{t}=\nabla \cdot(D(\nabla p+n \nabla \varphi)) & \text { in } & \Omega \times(0, \infty), \\
\Delta \varphi=n & \text { in } \quad \Omega \times(0, \infty), \\
(\nabla p+\nabla \varphi) \cdot \bar{\nu}=\varphi=0 & \text { on } \quad \partial \Omega \times(0, \infty), \\
n(0)=n_{0} \geq 0 & \text { in } \quad \Omega,
\end{array}
$$

where $\Omega \subset \mathbb{R}^{d}$ is a bounded domain and the pressure is self-similar, i.e.

$$
p(n, \theta)=\theta^{d / 2+1} P\left(n \theta^{-d / 2}\right) .
$$

Notice that due to (5) and a specific choice of the diffusion coefficient, [5], [1], [2],

$$
D=P^{\prime}
$$

the system (1)-(4) can be transformed to the following one:

$$
\begin{array}{rll}
n_{t}=\nabla \cdot\left(\theta P^{\prime 2} \nabla n+n P^{\prime} \nabla \varphi\right) & \text { in } & \Omega \times(0, \infty), \\
\Delta \varphi=n & \text { in } \quad \Omega \times(0, \infty), \\
\left(\theta P^{\prime 2} \nabla n+n P^{\prime} \nabla \varphi\right) \cdot \bar{\nu}=\varphi=0 & \text { on } & \partial \Omega \times(0, \infty), \\
n(0)=n_{0} \geq 0 & \text { in } & \Omega,
\end{array}
$$

2000 Mathematics Subject Classification: 35Q, 35K60, 82C21.

Key words and phrases: Chavanis-Sommeria-Robert model, Fermi-Dirac statistics, polytropic stars, nonlinear nonlocal parabolic-elliptic system.

The paper is in final form and no version of it will be published elsewhere. 
with a given positive continuous temperature $\theta=\theta(t)$ and the nonnegative density of the particles $n=n(x, t)$ and gravitationally induced potential $\varphi=\varphi(x, t)$ to be solved for.

Numerous pressure formulas coming from statistical mechanics of self-interacting particles have been collected in [3] including Maxwell-Boltzmann, Bose-Einstein, FermiDirac and polytropic distributions. We list below all of them in a series of examples but subsequently we shall focus on those $P$, which have the power of order $1+2 / d$ as the highest order term at infinity, i.e.,

$$
P(z)=p_{1} z^{1+2 / d}+R(z),
$$

with $R(z)=o\left(z^{1+2 / d}\right)$, as in examples 2 and 4 presented at the end of this section.

In [1] the authors proved the existence result for the specific choice of the diffusion parameter $D=P^{\prime}$ and density $p$ corresponding to the Fermi-Dirac statistics in the isothermal three-dimensional case $\theta=$ const (cf. Example 2 below). Moreover, the asymptotic behaviour with the possibility of the evolution towards steady states was addressed in [2] for nonisothermal case. The problem of existence of steady states was examined in [7].

In this paper we will prove some a priori estimates for the solutions of the problem (7)-(10), both for the density $n$ (Section 2) and for the temperature (Section 3) under the assumption that at $z=\infty$ the leading term of the $P(z)$ function is of the form $p_{1} z^{1+2 / d}$ as in (11). Thus, the polytropic and the Fermi-Dirac cases are relevant examples. These estimates cannot be derived from the paper [1] as the isothermal ( $\theta=$ const) entropy, used there to get $L^{1+2 / d}$ bounds for $n$, is no longer an entropy if we allow nonconstant temperature $\theta=\theta(t)$. Instead, in Section 2, we use the 'asymptotic energy' to get the desired estimate. Finally, we relegate the technical properties of the Fermi-Dirac model to Appendix.

The results presented here are also related to the problem of the existence of solutions to (7)-(10) in the microcanonical (nonisothermal) setting, i.e. with the given energy and the temperature to be determined so that the energy relation

$$
E=\frac{d}{2} \int_{\Omega} \vartheta^{d / 2+1} P\left(n \vartheta^{-d / 2}\right) d x+\frac{1}{2} \int_{\Omega} n \varphi d x=\text { const }
$$

is satisfied for $\vartheta=\theta$. This, however, is the subject of the forthcoming paper [9], and so is not extensively addressed herein. For the related steady states problem one can see [8]. In Section 3, we show in the main Theorem 3.2 that all the values of the energy attained at $t=0$ are admissible at $t>0$, i.e., could be attained at some $\vartheta=\vartheta(t)$, under the smallness assumption of mass $\int_{\Omega} n d x \ll 1$ which is crucial for the existence theorem in the microcanonical case. The existence result can be interpreted that the no gravothermal catastrophe occurs provided that the mass of the cloud of particles is relatively small compared to the energy. The details on the existence theorem are postponed to [9].

A related Keller-Segel model of chemotaxis phenomena with nonlinear diffusion but in the whole space was recently studied in [6], [10], [11]. Note that this model corresponds to the polytropic case and an analogous a priori estimate could be derived for the corresponding Keller-Segel model provided that the diffusion and transport coefficients have the required form. 
We end this section with a series of examples, in which the pressure has self-similar form (5).

ExAmple 1. For the Maxwell-Boltzmann distributions

$$
p_{\mathrm{MB}}(n, \theta)=\theta n,
$$

which follows after substitution in (5) $P_{M B}=I$. This classical Boltzmann relation leads to the linear Brownian diffusion term $\Delta n$ in (7) and, as such, will not be considered herein.

ExAmple 2. For the Fermi-Dirac distributions one has

$$
P_{F D}(z)=\frac{\mu}{d}\left(f_{d / 2} \circ f_{d / 2-1}^{-1}\right)\left(\frac{2}{\mu} z\right)
$$

implying, by (5),

$$
p_{\mathrm{FD}}(n, \theta)=\frac{\mu}{d} \theta^{d / 2+1}\left(f_{d / 2} \circ f_{d / 2-1}^{-1}\right)\left(\frac{2}{\mu} \frac{n}{\theta^{d / 2}}\right) .
$$

The function $f_{\alpha}$ denotes the Fermi integral of order $\alpha>-1$ defined by

$$
f_{\alpha}(z)=\int_{0}^{\infty} \frac{y^{\alpha} \mathrm{d} y}{\mathrm{e}^{y-z}+1}
$$

This leads to a nonlinear diffusion in (7). Properties of Fermi integrals (15) (convexity, asymptotics, etc.) relevant to study the system (1)-(4) are collected in $[1$, Sec. 2] and [2, Sec. 5], and our Appendix. In this case the leading term coefficient, being the limit of $P_{F D}(z) z^{-1-2 / d}$ as $z \rightarrow \infty$, equals $p_{1}=\frac{2}{d+2}\left(\frac{d}{\mu}\right)^{2 / d}$, where $\mu=\eta_{0} 2^{d / 2} G \sigma_{d}^{2}$ and $G$ is the gravitational constant, $\sigma_{d}$ the measure of a unit sphere in $\mathbb{R}^{d}$, and $\eta_{0}$ a bound on the density in the $(x, v)$ phase space. For the origins of the derivation of the simplified model (7)-(10) in the Fermi-Dirac case, see the argument at the end of Appendix.

EXAMPLE 3. An analogous construction is used to define the Bose-Einstein distributions whose properties, however, differ from those of Fermi-Dirac ones,

implying

$$
P_{B E}(z)=\frac{\mu}{d}\left(g_{d / 2} \circ g_{d / 2-1}^{-1}\right)\left(\frac{2}{\mu} z\right),
$$

$$
p_{\mathrm{BE}}(n, \theta)=\frac{\mu}{d} \theta^{d / 2+1}\left(g_{d / 2} \circ g_{d / 2-1}^{-1}\right)\left(\frac{2}{\mu} \frac{n}{\theta^{d / 2}}\right),
$$

which leads again to a nonlinear diffusion in (7). Here $g_{\alpha}$ denotes the Bose-Einstein integral of order $\alpha>-1$ (cf. [3, Sec. 2]) defined, for $z<0$, by

$$
g_{\alpha}(z)=\int_{0}^{\infty} \frac{y^{\alpha} \mathrm{d} y}{\mathrm{e}^{y-z}-1}
$$

In this case, however, due to the fact that, for $\alpha>0, G=\sup _{z<0} g_{\alpha}(z)$ is finite, $p_{\mathrm{BE}}$ could only be defined for $\frac{2}{\mu} \frac{n}{\theta^{d / 2}} \leq G$. Thus, since the asymptotic condition (11) is irrelevant here, this case is excluded from our further considerations.

EXAmple 4. Polytropes are classical equations of state of a gas with

$$
p_{1+\gamma}(n, \theta)=\kappa_{\gamma} \theta^{1-\gamma d / 2} n^{1+\gamma}
$$


with a polytropic constant $\kappa_{\gamma}>0$ and $0<\gamma<2 / d$ so that $\frac{\partial p}{\partial \theta}>0$, which is physically a natural condition. The limit value $\gamma=2 / d$ leads to the pressure

$$
p_{1+2 / d}(n, \theta)=\kappa_{2 / d} n^{1+2 / d}
$$

independent of $\theta$ and satisfies (11). The limit case $\gamma \searrow 0$ corresponds to the Boltzmann density-pressure relation (1). The polytropic relations define evolution equations with nonlinear diffusions as, e.g., in the porous media equation.

2. A priori estimates for the density. In this section we generalize some of the results obtained in [1], where the authors considered Fermi-Dirac density $P_{F D}$ and a constant temperature $\theta$, whereas here we treat more general pressure forms $P$ and allow a variable temperature $\theta=\theta(t)$.

First, note that, as a consequence of (3), total mass

$$
M=\int_{\Omega} n(x, t) d x
$$

is conserved during the evolution of the solution to the system (7)-(10).

Lemma 2.1. For any $2 \leq d \leq 4$ we have the estimate

$$
\left|\int_{\Omega} n \varphi d x\right| \leq C M^{1-2 / d} \int_{\Omega} n^{1+2 / d} d x .
$$

Proof. Apply the Sobolev-Gagliardo-Nirenberg and Hölder inequalities.

Now, we shall prove some a priori estimate for $L^{1+2 / d}$ norm of the density $n$. We will derive it directly from the equations (7)-(10).

Lemma 2.2. Assume that, for $P \in C^{1}$, (11) holds with $R$ satisfying additionally the global condition

$$
\left|R^{\prime}(z)\right| z^{1 / 2-1 / d} \leq B
$$

Define the 'asymptotic energy' by

$$
E^{a}(t)=\frac{d}{2} \int_{\Omega} p_{1} n^{1+2 / d} d x+\frac{1}{2} \int_{\Omega} n \varphi d x
$$

Then, there is a constant $C>0$ such that, for any fixed $T>0$ and any $t \in[0, T]$,

$$
\frac{d}{d t} E^{a}(t) \leq C \theta^{d / 2} \int_{\Omega}|\nabla \varphi|^{2} d x
$$

Proof. Let $t \in[0, T]$ and recall that both $P$ and $P^{\prime}$ are continuous functions of $n \theta^{-d / 2}$. Now, we multiply (7) by $\frac{d}{2} n^{2 / d}$ and integrate over $\Omega$ to obtain

$$
\frac{d^{2}}{2(d+2)} \frac{d}{d t} \int_{\Omega} n^{1+2 / d} d x=-\theta \int_{\Omega} P^{\prime 2}|\nabla n|^{2} n^{2 / d-1} d x-\int_{\Omega} P^{\prime} n^{2 / d} \nabla n \cdot \nabla \varphi d x .
$$

Similarly, multiplying (7) by $A \varphi$, we get

$$
\frac{A}{2} \frac{d}{d t} \int_{\Omega} n \varphi d x=-A \int_{\Omega} P^{\prime} n|\nabla \varphi|^{2} d x-A \theta \int_{\Omega} P^{\prime 2} \nabla n \cdot \nabla \varphi d x .
$$


Summing up the above equalities and using the following Hölder inequality:

$$
\begin{aligned}
& \left|\int_{\Omega} P^{\prime} \theta^{1 / 2} n^{1 / d-1 / 2} \nabla n \cdot \nabla \varphi\left(\theta^{-1 / 2} n^{1 / d+1 / 2}+A P^{\prime} \theta^{1 / 2} n^{-1 / d+1 / 2}\right) d x\right| \\
& \leq \int_{\Omega}\left\{P^{\prime 2} \theta n^{2 / d-1}|\nabla n|^{2}+\frac{1}{4}|\nabla \varphi|^{2}\left(\theta^{-1 / 2} n^{1 / d+1 / 2}+A P^{\prime} \theta^{1 / 2} n^{-1 / d+1 / 2}\right)^{2}\right\} d x
\end{aligned}
$$

we arrive, taking $A(d+2) p_{1}=d$, at

$$
\frac{d}{d t} E^{a}(t) \leq \frac{1}{4 A} \int_{\Omega}|\nabla \varphi|^{2}\left(A P^{\prime} \theta^{1 / 2} n^{-1 / d+1 / 2}-\theta^{-1 / 2} n^{1 / d+1 / 2}\right)^{2} d x .
$$

This yields the claim with $C=\frac{d B^{2}}{4(d+2) p_{1}}$, by the assumption (22) together with (11) implying $P^{\prime}(z)=\left(p_{1}(d+2) / d\right) z^{2 / d}+R^{\prime}(z)$.

REMARK. Note that Lemma 2.2 holds in the polytropic case with $R(z)=0$ as well as in the Fermi-Dirac case. Indeed, from Lemma 4.2 in Appendix it follows that $R^{\prime}(z)=$ $\mathcal{O}\left(z^{-2 / d}\right)$ at $z=\infty$ and $\left|R^{\prime}(z)\right| \leq B$ at $z=0$. This implies $\left|R^{\prime}(z)\right| \leq B z^{1 / d-1 / 2}$, for $2 \leq d \leq 6$.

REMARK. It should be noted that for the polytropic case Lemma 2.2 implies the dissipation of the energy, since in this case $E^{a}=E$ defined by (12). Therefore this energy conservation postulate makes no sense in this case. Note also the disappearance of the temperature in the formula (19).

REMARK. The name 'asymptotic energy' used here for $E^{a}$ can be understood, under monotonicity assumption (eg. (33)), as the property $E^{a}(t)=\lim _{\vartheta \rightarrow 0^{+}} E(t)$.

Applying the estimate (21) from Lemma 2.1 and integrating (24) from Lemma 2.2 allows us to derive the following proposition.

Proposition 2.3. Under the assumptions of Lemma 2.2, for $C M^{1-2 / d}<d p_{1}$, the integral form of the growth condition reads

$$
0 \leq E^{a}(t) \leq E^{a}(0) \exp (C(t))
$$

where $C(t)=C_{M} \int_{0}^{t} \theta^{d / 2}(s) d s$ with $C_{M}=\frac{2}{d p_{1} C^{-1} M^{2 / d-1}-1}$.

Proof. Applying the estimate (21) we get, for $C M^{1-2 / d}<d p_{1}$,

$$
\theta^{d / 2} \int_{\Omega}|\nabla \varphi|^{2} d x \leq C_{M} \theta^{d / 2}\left(\frac{d}{2} \int_{\Omega} p_{1} n^{1+2 / d} d x-\frac{1}{2} \int_{\Omega}|\nabla \varphi|^{2} d x\right),
$$

where $C_{M}=\frac{2}{d p_{1} C^{-1} M^{2 / d-1}-1}$. Applying this estimate to (24) yields the claim.

Combining Lemma 2.1 with the above proposition, we are ready to obtain an $L^{1+2 / d}$ estimate for the density $n$.

COROLlary 2.4. Assume that (11) holds with the lower order term satisfying (22) and take sufficiently small mass $M^{1-2 / d} \ll d p_{1}$. Then

$$
\int_{\Omega} n^{1+2 / d} d x \leq B_{M} E^{a}(0) \exp (C(t)),
$$

where $C(t)=C_{M} \int_{0}^{t} \theta^{d / 2}(s) d s$ with some constants $B_{M}, C_{M}$. 
Owing to Corollary 2.4, an $L^{2}$-estimate is also available for $n$. This will be the claim of the next lemma which has its origins in the paper [1], where $d=3, P=P_{F D}$ and $\theta=$ const. Here the dependence on variable $\theta=\theta(t)$, which was irrelevant in [1], is meticulously examined and any dimension $d \geq 2$ is considered $\left(2 \leq d \leq 6\right.$ for $\left.P_{F D}\right)$.

LEMMA 2.5. Let $T>0,2 \leq d \leq 4$ and assume (11), (22),

$$
P^{\prime}(z) \geq p_{0}
$$

and

$$
p_{1} z^{1+2 / d} \leq P(z) \leq p_{2}\left(z^{1+2 / d}+1\right) .
$$

Then, there is a constant $A$, coming from Sobolev embedding, and a constant $C$ depending on the initial data, bounds on $\vartheta \in[a, b]$ and $T$ such that, for $t \in[0, T]$ and $A M^{4 / d}<1$,

$$
|n(t)|_{2}+a p_{0}^{2}\left(1-A M^{4 / d}\right) \int_{0}^{t}|\nabla n|_{2}^{2} d s \leq C .
$$

Proof. Let $t \in[0, T]$ and multiply (7) by $2 n$ and integrate over $\Omega$ to obtain

$$
\frac{d}{d t}|n(t)|_{2}^{2}+2 \theta \quad \int_{\Omega} P^{\prime 2}|\nabla n|^{2} d x=-2 \int_{\Omega} n P^{\prime} \nabla n \cdot \nabla \varphi d x .
$$

Next, we have

$$
2\left|\int_{\Omega} n P^{\prime} \nabla n \cdot \nabla \varphi d x\right| \leq \theta \int_{\Omega} P^{\prime 2}|\nabla n|^{2} d x+\frac{1}{\theta} \int_{\Omega} n^{2}|\nabla \varphi|^{2} d x
$$

by the Young inequality, whence

$$
\frac{d}{d t}|n(t)|_{2}^{2}+\theta \quad \int_{\Omega} P^{\prime 2}|\nabla n|^{2} d x \leq \frac{1}{\theta} \int_{\Omega} n^{2}|\nabla \varphi|^{2} d x .
$$

From (28) we get $\theta^{d / 2+1} P\left(n \theta^{-d / 2}\right) \geq p_{1} n^{1+2 / d}$ and using the Hölder inequality and Sobolev embeddings we obtain, setting $\|\cdot\|_{*}$ a norm in $H_{0}^{1}(\Omega)$,

$$
\begin{gathered}
\int_{\Omega} n^{2}|\nabla \varphi|^{2} \leq\left|n^{1+2 / d}\right|_{\frac{2 d}{d-2}}^{\frac{2 d}{d+2}}|\nabla \varphi|_{\frac{d+2}{2}}^{2} \leq\left|n^{1+2 / d}\right|_{\frac{2 d}{d-2}}^{\frac{2 d}{d+2}}|n|_{\frac{d(d+2)}{3 d+2}}^{2} \\
\leq\left|\theta^{d / 2+1} P\right|_{\frac{2 d}{d-2}}^{\frac{2 d}{d+2}}\left|n^{1+2 / d}\right|_{\frac{2 d}{d-2}}^{\frac{4}{d+2}} M^{4 / d} \leq A\left\|\theta^{d / 2+1} P\right\|_{*}^{2} M^{4 / d} .
\end{gathered}
$$

This estimate applied to the right-hand side of (30), by (28), yields

$$
\frac{d}{d t}|n(t)|_{2}^{2}+a p_{0}^{2}\left(1-A M^{4 / d}\right) \int_{\Omega}|\nabla n|^{2} d x \leq \frac{A}{\theta}\left|\theta^{d / 2+1} P\right|_{1}^{2} M^{4 / d},
$$

which ends the proof if we integrate the above inequality with respect to time and apply (28) and Corollary 2.4.

REMARK. Note that after the modification of the proof the above theorem is also valid for $d=2$. Note also that while assumption (28) is satisfied both for Fermi-Dirac and polytropic model, condition (27) holds only for $P_{F D}$ and is violated for polytropes in the neighbourhood of 0 .

REMARK. Lemma 2.5 implies also an $L^{\infty}$ bound by a standard bootstrap argument, cf. $[1,9]$. Those $L^{\infty}$ estimates are rather crude, i.e. insensitive to the signs of the terms 
involved, differently from the case $L^{1+2 / d}$ or $L^{2}$ estimates presented above which took advantage of the negative sign of the diffusion term.

3. Fixed energy problem. If we postulate conservation of the energy (12) some a priori bounds follow directly as was done e.g. in [1] in the Fermi-Dirac case and in [3] and [4] in more general case. Recall the following version of energy estimates (for the proof see [4, Lemma 3.1]) valid in dimensions $d=2,3$.

Lemma 3.1. Let $\nu=4 /(d(4-d))$. Provided that $P(s) \geq p_{1} s^{1+2 / d}$, for any $\frac{d}{2} p_{1}>\varepsilon>0$ and all $s \geq 0$ the following estimate holds:

$$
E+C M^{1+\nu} \geq \max \left\{\varepsilon \int_{\Omega} n^{1+2 / d} d x, \int_{\Omega}|\nabla \varphi|^{2} d x\right\} .
$$

Finally, for each $0<\varepsilon<d / 2$

$$
E \geq \varepsilon \int_{\Omega} \vartheta^{d / 2+1} P\left(n \vartheta^{-d / 2}\right) d x+\left|\int_{\Omega} n \varphi d x\right|-C M^{1+\nu} .
$$

Note that this lemma does not imply the estimates from Section 2 which were obtained without energy conservation claim directly from the equations (7)-(10). Corollary 2.4 is of great importance not only for further improvement of the integrability and regularity properties of the density (cf. Section 2 and [9]) but to establish what is the range of the energy (12). This issue was not addressed in [4] where under physically acceptable property of the pressure: $\frac{\partial p}{\partial \vartheta}>0$ expressed as

$$
P(z) z^{-1-2 / d} \searrow p_{1}>0
$$

the authors proved only the uniqueness of the temperature $\vartheta$ defined by the energy formula (12). Now, in the following theorem, we are going to show that all positive values of the energy are attained for some value $\vartheta(t)=\vartheta$ (we fix $t>0$ ).

Theorem 3.2. Assume that (11), (22), (27) and (33) hold. Then the temperature operator $\mathcal{T}: \theta \rightarrow \vartheta$ is formally well defined by (12) for $M \ll 1$ and all the values of the energy $E$ admissible at $t=0$. Moreover, for $d=2,3, \nu=4 /(d(4-d))$ and some postitive constants $B, C$, it has to satisfy

$$
E<B M^{1+2 / d}-C M^{1+\nu} .
$$

Proof. We are going to show that $E(\vartheta)$ can be defined for $\vartheta=\vartheta(t)>0$, with fixed $t>0$, by $(12)$. In fact, it maps $(0, \infty)$ onto interval $\left(E^{a}, \infty\right) \ni E$. To this end, define

$$
(2 / d) F(\vartheta)=\int_{\Omega} p d x-\int_{\Omega} p_{1} n^{1+2 / d} d x .
$$

Then, by Proposition 2.3 and the identity $E^{a}=E(\vartheta)-F(\vartheta)$, for any positive $E>0$ attained initially at $t=0$

$$
E(\vartheta) \leq F(\vartheta)+\left(E-F\left(\vartheta_{0}\right)\right) \exp (C(t))
$$

but due to assumption (33)

$$
F\left(\vartheta_{0}\right)>F(0)=0 .
$$


Since $F(\vartheta) \rightarrow 0$ as $\vartheta \rightarrow 0^{+}$, we have for small $\vartheta$

$$
E(\vartheta)<E
$$

provided $M$ is small enough to guarantee, by (2.1), that

$$
\exp (C(t))<\frac{E}{E-F\left(\vartheta_{0}\right)} .
$$

Moreover, by (27), $E(\vartheta) \geq(d / 2) M p_{0} \vartheta+(1 / 2) \int_{\Omega} n \varphi d x$ yields $E(\vartheta) \rightarrow \infty$ as $\vartheta \rightarrow \infty$. The existence of positive $\vartheta$ such that the given, positive energy $E$ is attained

$$
E(\vartheta)=E
$$

is implied by the continuity with respect to $\vartheta$ which proves the claim. Finally, (34) is an easy consequence of (31) and (28).

REMARK. Note that all the assumptions are satisfied for the Fermi-Dirac pressure, the superlinearity assumption fails for polytropes, which is natural since, as a matter of fact, the temperature $\vartheta$ disappears in the formula (12) for the polytropic pressure.

Finally, we shall show some a priori estimates for the fixed points of the temperature operator $\mathcal{T}$ in the case $P$ satisfies (11). First, we recall after [3] that the functional $\mathcal{W}$

$$
\mathcal{W}=\int_{\Omega}\left(n H-\left(\frac{d}{2}+1\right) P \theta^{d / 2}\right) d x
$$

is a Lyapunov functional for the problem (7)-(8) under the energy constraint (12). The function $H(z)$ depending on $z=n \theta^{-d / 2}$ is a primitive of $P^{\prime}(z) / z$. However, due to the assumption (11) the leading terms in (37) of order $1+2 / d$ cancel out, and lower order terms do not provide any better a priori estimates for the density $n$ than (31). On the other hand, the entropy can be used to get a priori bounds for the temperature as was done in [3]. The authors assumed there, for negative initial values of the entropy, that

$$
\liminf _{z \rightarrow \infty}(H(z)-(d / 2+1) P(z) / z)>\mathcal{W}(0) / M .
$$

If (11) is fulfilled then as we announced the highest order terms cancel and, if the limit exists

$$
\begin{array}{r}
\lim _{z \rightarrow \infty} \frac{H(z)-(d / 2+1) P(z)}{z}=\lim _{z \rightarrow \infty}\left(H(z)-\frac{d}{2} z H^{\prime}(z)\right) \\
=\lim _{z \rightarrow \infty}\left(G(z)-(d / 2) z G^{\prime}(z)\right) \stackrel{\text { df }}{=} G_{0}
\end{array}
$$

where

$$
H(z)=h_{1} z^{2 / d}+G(z)
$$

with $G^{\prime}(z)=o\left(z^{2 / d}-1\right)$, we are left with the analysis of the lower order term $G^{\prime}(z)=$ $g_{1} z^{\beta}+o\left(z^{\beta}\right)$ with some $\beta<2 / d-1$. Namely, if $\beta<1$ then $G_{0}=0$ in (39), e.g. for the Fermi-Dirac model in $d=2$. Otherwise, if $\beta \in[-1,2 / d-1)$ then the important factor is the sign of $g_{1}$ which has to be positive, and indeed is, e.g. $g_{1}=1-2 / d$ in the Fermi-Dirac case for $d \geq 3$, to imply $G_{0}=-\infty$ and thus to guarantee (38). 
4. Appendix on Fermi-Dirac model. To prove the asymptotics for the Fermi-Dirac pressure $P_{F D}$ we need to recall some asymptotic properties of Fermi functions. In [2, Lemma 5.1] substitute $z=-\log (\lambda)$ and $f_{\alpha}(z)=I_{\alpha}\left(e^{-z}\right)$ to get the following

LEMMA 4.1. The following asymptotic relations hold as $z \rightarrow \infty$ :

$$
f_{\alpha}(z)-\frac{z^{\alpha+1}}{\alpha+1}=\mathcal{O}\left(z^{\alpha-1}\right)
$$

for each $\alpha \geq 0$, while for each $\alpha>-1$

$$
z^{-\alpha}\left\{f_{\alpha}(z) z-\frac{\alpha+2}{\alpha+1} f_{\alpha+1}(z)\right\} \rightarrow-\frac{\pi^{2}}{3}
$$

Moreover, we have the iterative relation for the derivatives

$$
f_{\alpha}^{\prime}(z)=\alpha f_{\alpha-1}(z)
$$

Now the announced asymptotic result follows.

Lemma 4.2. For the Fermi-Dirac pressure form $P_{F D}$ we have at $z=\infty$

$$
R_{F D}^{\prime}(z)=\mathcal{O}\left(z^{-2 / d}\right)
$$

and

$$
P_{F D}(z)=p_{1} z^{1+2 / d}+\mathcal{O}\left(z^{1-2 / d}\right) .
$$

where $p_{1}=\frac{2}{d+2}(d / \mu)^{2 / d}$. Moreover, at $z=0$, we have

$$
R_{F D}^{\prime}(z)=\mathcal{O}(1) \text {. }
$$

Proof. To prove (44), we need to apply Lemma 4.1. First, using formula (13) gives, for $w=f_{d / 2-1}^{-1}(2 z / \mu)$,

$$
P_{F D}^{\prime}(z)=\frac{f_{d / 2-1}(w)}{f_{d / 2-1}^{\prime}(w)}
$$

We want to prove that

$$
\left(P_{F D}^{\prime}(z)-p_{1}(1+2 / d) z^{2 / d}\right) z^{2 / d}=\mathcal{O}(1)
$$

or equivalently, recalling that $2 z=\mu f_{d / 2-1}(w)$ and (41), the boundedness of

$$
w\left(\frac{f_{2 / d-1}(w)}{(d / 2-1) f_{d / 2-2}(w)}-\frac{2 w}{d}+\frac{2 w}{d}-p_{1}\left(1+\frac{2}{d}\right)\left(\frac{\mu}{2} f_{d / 2-1}(w)\right)^{2 / d}\right) .
$$

In the above formula the first two terms, due to (41)-(42), converge at $\infty$ to some constant. As for the next two, more detailed calculations are required. To this end we express

$$
\frac{2 w^{2}}{d}-p_{1} w\left(1+\frac{2}{d}\right)\left(\frac{\mu}{2} f_{d / 2-1}(w)\right)^{2 / d}
$$

as

$$
\left(w^{d / 2}-A^{d} f_{d / 2-1}(w)\right) \frac{2 w\left(w^{1 / 2}+A f_{d / 2-1}(w)^{1 / d}\right)}{d \sum_{k=0}^{d-1} w^{k / 2} f_{d / 2-1}(w)^{(d-1-k) / d} A^{d-1-k}}
$$

with $A^{2}=p_{1}(1+d / 2)(\mu / 2)^{2 / d}$ and the first term of order $\mathcal{O}\left(w^{d / 2-2}\right)$ and the second one (the fraction) like $\mathcal{O}\left(w^{2-d / 2}\right)$. Thus we have just obtained the claimed boundedness. 
Moreover,

$$
P_{F D}(z)=p_{1} z^{1+2 / d}+\mathcal{O}\left(z^{2 / d(d / 2-1)}\right)
$$

follows, by (41), from

$$
f_{d / 2}(z)=\frac{2}{d+2} z^{d / 2+1}+\mathcal{O}\left(z^{d / 2-1}\right)
$$

and

$$
2 z / \mu=2 / d\left(f_{d / 2-1}^{-1}(2 z / \mu)\right)^{d / 2}+\mathcal{O}\left(\left(f_{d / 2-1}^{-1}(2 z / \mu)\right)^{d / 2-2}\right) .
$$

The last claim of this lemma (46) follows from the asymptotic behaviour of the Fermi functions. Namely, from [2, Section 5] it follows that

$$
f_{\alpha}(z) \exp (z)=\mathcal{O}(z)
$$

at $z=0$ which guarantees (46), and ends the proof of this lemma.

Now, we shall show how (7)-(10) could be derived, in the Fermi-Dirac case, from (1)-(4) under the assumption (5) with a particular diffusion coefficient. Recall that both in [1] and [5] the authors used

$$
D(\lambda)=\frac{-I_{d / 2-1}(\lambda)}{\lambda I_{d / 2-1}^{\prime}(\lambda)}
$$

where $I_{\alpha}\left(e^{-z}\right)=f_{\alpha}(z)$ is another version of the Fermi function and

$$
\lambda=I_{d / 2-1}^{-1}\left(\frac{2 n}{\mu \theta^{d / 2}}\right) .
$$

Using the recurrence derivation property (43) (cf. also [2, Section 5] and [7, Lemma 1.1]) and (47), we recover claimed relation $D=P^{\prime}$.

Moreover, it should be noted that in [1] and [2] the authors used the following notation $F^{\prime}=P^{\prime 2}, V=n P^{\prime}$. Note that $D$ should be defined exactly as in (48), which might differ throughout these papers up to an inessential constant.

Acknowledgements. The preparation of this note was partially supported by the European Commission Marie Curie Host Fellowship for the Transfer of Knowledge "Harmonic Analysis, Nonlinear Analysis and Probability" MTKD-CT-2004-013389.

\section{References}

[1] P. Biler, Ph. Laurençot and T. Nadzieja, On an evolution system describing self-gravitating Fermi-Dirac particles, Adv. Differential Equations 9 (2004), 563-586.

[2] P. Biler, T. Nadzieja and R. Stańczy, Nonisothermal systems of self-interacting FermiDirac particles, in: Nonlocal Elliptic and Parabolic Problems, P. Biler, G. Karch and T. Nadzieja (eds.), Banach Center Publications 66, Inst. Math. Polish Acad. Sci., Warszawa, 2004, 61-78.

[3] P. Biler and R. Stańczy, Parabolic-elliptic systems with general density-pressure relations, in: Variational Problems and Related Topics, M. Misawa and T. Suzuki (eds.), Sūrikaisekikenkyūsho Kōkyūroku 1405 (2004), 31-53. 
[4] P. Biler and R. Stańczy, Nonlinear diffusion models for self-gravitating particles, in: Free Boundary Problems Theory and Applications, I. N. Figueiredo, J. F. Rodrigues and Lisa Santos (eds.), International Series of Numerical Mathematics 154, Birkhäuser, Basel, 2006.

[5] P.-H. Chavanis, C. Rosier and C. Sire, Thermodynamics of self-gravitating systems, Phys. Rev. E 66 (2002), 036105.

[6] S. Luckhaus and Y. Sugiyama, Large time behavior of solutions in subcritical cases to degenerate Keller-Segel systems, Mathematical Modelling and Numerical Analysis 40 (2006), 597-621.

[7] R. Stańczy, Steady states for a system describing self-gravitating Fermi-Dirac particles, Differential Integral Equations 18 (2005), 567-582.

[8] R. Stańczy, Self-attracting Fermi-Dirac particles in canonical and microcanonical setting, Mathematical Methods in Applied Sciences 28 (2005), 975-990.

[9] R. Stańczy, On an evolution system describing self-gravitating particles in microcanonical setting, submitted.

[10] Y. Sugiyama and H. Kunii, Global existence and decay properties for a degenerate KellerSegel model with a power factor in drift term, J. Differential Equations 227 (2006), 333364.

[11] Y. Sugiyama, Global existence and finite time blow up for some degenerate Keller-Segel model, in: Variational Problems and Related Topics, Y. Naito and T. Suzuki (eds.), Sūrikaisekikenkyūsho Kōkyūroku 1464 (2005), 40-57. 
